\title{
Desde una mirada decolonial y feminista hacia la Educación Sexual Integral: La Dama de Cao
}

From a decolonial and feminist perspective to a Comprehensive Sex Education: The Lady of Cao

\author{
Antonella Fanucci ${ }^{1}$ \\ Bárbara Noriega ${ }^{2}$
}

\section{Resumen}

El objetivo de este trabajo es presentar la necesidad de contenidos pedagógicos en torno a las mujeres de comunidades indígenas en la enseñanza argentina. Por esto, nos parece que la investigación y divulgación del hallazgo arqueológico, la Dama de Cao de la historia del Perú Antiguo, colabora para trabajar los lineamientos de la ley 26.150 de nuestro país en torno al ciclo básico. Desde nuestra área académica, la historia, proponemos el caso mochica porque consideramos que permite amplificar la representación de la mujer indígena y el rol de la mujer en el pasado en clave latinoamericana, desde una mirada decolonial. A partir del caso esbozado, invitamos a una lectura que busca re-construir y re-significar la importancia de las mujeres indígenas para repensar los interrogantes en torno a nuestro territorio y a nuestra identidad latinoamericana.

Palabras claves: Dama de Cao - educación - perspectiva de género - decolonial

\section{Abstract}

The aim of this paper is to present the need for educational content about women from indigenous communities in Argentine education. For this reason, we believe that the research and dissemination of the archaeological find, the Lady of Cao of the history of Ancient Peru, collaborates to work on the guidelines of law 26.150 of our country regarding the basic cycle. From our academic area, history, we propose the Mochica case because we consider that it allows us to amplify the representation of the indigenous woman and the role of women in the past in a Latin American key, from a decolonial perspective. From the case outlined, we invite a reading that

\footnotetext{
Recibido: 30 de marzo de 2020 Aceptado: 4 de julio de 2020 Publicado: 10 de julio de 2020

${ }^{1}$ Estudiante de la carrera de Historia de la Universidad Nacional de Rosario (FHUMYAR-UNR), Rosario, Argentina. Correo electrónico: antofanu97@gmail.com.

${ }^{2}$ Estudiante de la carrera de Historia de la Universidad Nacional de Rosario (FHUMYAR-UNR), Rosario, Argentina. Correo electrónico: barbaranoriega330@gmail.com
} 
seeks to re-construct and re-signify the importance of indigenous women in order to rethink the questions surrounding our territory and our Latin American identity.

Keywords: Lady of Cao- education- gender perspective-decolonial

\section{Introducción}

A partir del presente repensamos la falta de contenido de las historias de las mujeres indígena dentro del ámbito educativo, haciendo énfasis en el ciclo básico argentino. Consideramos la necesidad de replantearnos: ¿por qué se invisibiliza a las mujeres indígenas? ¿Cómo aparecen en los contenidos educativos de nuestra provincia? ¿Según el marco normativo santafesino qué sucede con la Ley de Educación Sexual Integral? ¿En qué medida el hallazgo de la Dama de Cao nos ayuda a enseñar historia con perspectiva de género en instituciones educativas?

A partir de estos interrogantes invitamos a reflexionar los nuevos descubrimientos, interpelando lo dado, pudiendo repensar y comprender no solo problemáticas del pasado, sino también del presente ámbito educativo. Desde la historia, pensamos que es innovador repensar la forma de enseñanza en torno a nuevos hallazgos, sobre todo incorporando una perspectiva de género que invita a reflexionar el pasado en articulación con la enseñanza educativa desde una perspectiva que contribuya a los lineamientos de la Ley de Educación Sexual Integral.

La perspectiva desde la cual buscamos interpelar es a través del desarme del discurso eurocéntrico y androcéntrico como un dispositivo colonizador del saber. Es por ello, que analizamos el ámbito pedagógico a través del caso del área andina, que no es el único. En nuestro territorio abundan ejemplos que son igual de importantes para problematizar en la enseñanza. A su vez, es una buena forma de deconstruir los márgenes nacionales y rescatar una noción presente en gran parte de las comunidades originarias con el respeto histórico que requiere la interpretación de su pasado social y político.

A partir de nuestro presente en torno a la enseñanza actual, creemos que el conocimiento de los hechos pasados puede adquirir una nueva vida como emblema de luchas contemporáneas por y para la emancipación y la igualdad.

\section{El fin del colonialismo sin fin: ¿Cómo combatimos el eurocentrismo?}

Denominamos este subtítulo teniendo en cuenta la interpretación del sociólogo Boaventura de Sousa Santos (2010) ya que nos invita a reflexionar acerca de que el fin del colonialismo político y las independencias americanas no significaron realmente su fin sino que muchas de nuestras prácticas teóricas y culturales, en relación con las 
mentalidades y subjetividades, continúan impregnadas de una lógica eurocentrista. En este sentido,

el pensamiento moderno occidental avanza operando sobre líneas abismales que dividen lo humano de lo subhumano de tal modo que los principios humanos no quedan comprometidos por prácticas inhumanas. Las colonias proveyeron un modelo de exclusión radical que prevalece hoy en día en el pensamiento y práctica occidental moderna como lo hicieron durante el ciclo colonial. Hoy como entonces, la creación y la negación del otro lado de la línea son constitutivas de los principios y prácticas hegemónicas (Santos, 2010, p.36).

Entonces podemos interrogarnos por el tipo de historia que se produce, se aprende y se enseña, la noción que se tiene de la alteridad ya que se construyó un otro bárbaro, incivilizado y lejano que no merecía contar su historia ni pertenecer a la sociedad. Esto se nos presenta como la historia, cuando en realidad es un fragmento, un recorte intencional desde donde se nos ha vetado gran parte de la historia de nuestros orígenes indígenas. Se nos enseña como propia una historia que en realidad es ajena y europea. De este modo, nuestro punto de partida es el desarme del discurso eurocéntrico “(...) que piensa y organiza a la totalidad del tiempo y del espacio, a toda la humanidad, a partir de su propia experiencia, colocando su especificidad histórico-cultural como patrón de referencia superior y universal" (Lander, 2000, p.24).

En palabras del sociólogo brasilero,

El privilegio epistemológico que la ciencia moderna se concede a sí misma, pues, el resultado de la destrucción de todos los conocimientos alternativos que podrían venir a enjuiciar ese privilegio. En otras palabras, el privilegio epistemológico de la ciencia moderna es producto de un epistemicidio. La destrucción del conocimiento no es un artefacto epistemológico sin consecuencias, sino implica la destrucción de prácticas sociales y la descalificación de agentes sociales que operan de acuerdo con el conocimiento enjuiciado (Santos, 2009, p.81).

Existió y existe ese epistemicidio que menciona Santos porque el genocidio no solo apuntó a la desaparición física de los pueblos indígenas sino a borrar sus huellas culturales y materiales y priorizó una sola forma de conocer. Es necesario situar históricamente este recorte intencionado, su dimensión y expansión hasta la actualidad donde la producción de la ciencia se cimentó bajo una pretensión de neutralidad y objetividad que priorizo el logos europeo como lo verdadero. Este proceso está 
fuertemente vinculado al nacimiento del capitalismo en donde la subordinación de los márgenes al centro-eje comercial y político impidió y puso trabas al desarrollo y crecimiento de aquellos considerados inferiores.

\section{Historia y género: ¿cómo combatimos el androcentrismo?}

Dentro de las ciencias sociales la historia, como otras disciplinas, nace a desde una perspectiva androcéntrica que prioriza las experiencias y miradas de los hombres en detrimento de las mujeres y construye una mirada única y universal. La historia como ciencia, además, surge enlazada al positivismo. Esta corriente, prioriza la objetividad, las fuentes escritas públicas, lo político, militar o diplomático, es decir, áreas históricamente ocupadas por el quehacer masculino de elite. Sin embargo, a partir del siglo XX, con la escuela de Annales en Francia la historia transformó su objeto de estudio y sus métodos. Comenzaron a considerarse otras fuentes y a pensar a la historia como problema.

(...) No fue sino hasta la década del 70 que las mujeres aparecen en escena, cuando la lucha de los movimientos sociales empieza a tener impacto en el ámbito académico. Fue la categoría género la herramienta fundamental que permitió incorporar la diferencia sexual como categoría de análisis. A partir de ello se fueron superando esencialismos biologicistas lo que permitió pensar en la heterogeneidad de la experiencia de las mujeres lejos de la idea de que la historia de las mujeres era la historia de víctimas de dominación, abriendo así el camino para analizar las causas de esa dominación (Argiroffo y Escalona, 2016, p.15-16).

De este modo, la categoría no es solamente política sino también académica en tanto nos permite complejizar los procesos sociales, donde cabe no sólo preguntarse por las mujeres sino también por las relaciones sociales para comprender a los sujetos históricos e interpretar su pasado y presente. Este concepto involucra las diversas áreas que componen una sociedad y las relaciones entre los distintos géneros que las constituyeron y construyeron, “(...) no alcanza con describir la experiencia de las mujeres, sino que es menester articularlas con un orden social determinado. El género es una categoría relacional" (Argiroffo y Escalona, 2016, p.15-16).

En este sentido, podemos sumar las interpretaciones de 1985 de la historiadora Joan Wallach Scott acerca de la definición de género como categoría 
histórica y analítica, ampliando nuestras conclusiones teóricas en relación con la construcción científica.

Género parece haberse convertido en una palabra particularmente útil a medida que los estudios sobre el sexo y la sexualidad han proliferado, porque ofrece un modo de diferenciar la práctica sexual de los roles sociales asignados a mujeres y hombres. Si bien los estudiosos reconocen la conexión entre sexo y (lo que los sociólogos de la familia llamaron) "roles sexuales", no asumen una relación sencilla y directa. El uso de género pone de relieve un sistema completo de relaciones que puede incluir el sexo, pero no está directamente determinado por el sexo o es directamente determinante de la sexualidad (Scott, 1990, p.7).

A lo dicho nos interesa incorporar la perspectiva de los feminismos decoloniales que nos ayuda a pensar la realidad de las mujeres indígenas que han sido despojadas de la historia por no ser europeas.

(..) Valoramos el concepto de género; sin embargo -al igual que los feminismos decoloniales- criticamos la homogeneización de la experiencia de las mujeres desde un molde construido por y para europeas, blancas, heterosexuales. Por ello, entendemos primordial realizar una intersección categorial de raza, clase, género y sexualidad, para aproximarse de forma integral a las consecuencias contextuales del colonialismo (Contreras y Cristoffanini, 2016, p.154).

De este modo, entendemos que al igual que la construcción cultural sexogénero y la de etnia-raza se creó una socialización diferente entre hombres-mujeres, blancos-indígenas; se construyó un otro que hoy es imprescindible visibilizar. Su experiencia en la historia como forma de poner en jaque las bases de la modernidad y del progreso en el proyecto colonial y volvió significativas otras huellas del pasado para poder encontrar lo que las mujeres dijeron de sí mismas, de los acuerdos y resistencias ante los modelos hegemónicos.

Desde nuestra perspectiva el caso mochica de la Dama de Cao permite cuestionar y deconstruir sobre cómo la modernidad construyó un binomio sexogénero particular y a su vez una desigualdad en detrimento de las mujeres que favorece a un orden político, económico y social, tanto en el ámbito público como privado.

En consecuencia, la ciencia se desarrolló desde una mirada androcéntrica y eurocéntrica y dentro de ella la diciplina histórica tuvo una incapacidad para cuestionar y problematizar el sistema sexo-género. Durante un largo proceso de desarrollo científico que influyó en la sociedad en general y en las formas en que se transmitió este conocimiento en particular se ejerció una influencia en las mentalidades, los cuerpos, que permeó todas las áreas de nuestra cosmovisión. 
Desde este punto en la actualidad las instituciones educativas tienen como desafío incorporar los avances científicos en torno a la categoría de género, no solo construir una historia que contemple las experiencias de las mujeres y las relaciones de género sino también deconstruir la categoría universal de mujer.

A su vez, dentro del colectivo indígena de mujeres también se generan particularidades, alianzas y tensiones propias de su espacio y contexto; se construyen distintas identidades y por lo tanto se da lugar a diferentes realidades. Es necesario pensar al colectivo de mujeres indígenas como un grupo heterogéneo donde cada comunidad tiene sus propias luchas y donde incluso se generan experiencias y prácticas diferentes con relación a las distintas comunidades y dentro de ellas.

Así mismo consideramos que a pesar de esta heterogeneidad y de la investigación que depara no creemos sea per se motivo de discusión ya que todos los grupos sociales, indígenas o no, son heterogéneos. Nos interesa esta diversidad o desigualdad en tanto se halla en articulación con una posición social, económica, política o demás perspectivas que analizan las ciencias sociales.

\section{Educación y currículum}

Para complejizar el análisis de la enseñanza en el ciclo básico, en particular a nivel regional, entendemos que es primordial comprender algunos problemas que se dan entorno al currículum que nos obliga a reconceptualizarlo. Debemos crear una apertura de aspectos a considerar, comprender y observar los elementos del currículum como categorías a desarrollar y no acabadas.

A modo de esbozar una definición tenemos en cuenta que el currículum implica la síntesis de elementos culturales (De Alba, 1995). En este sentido, el concepto de síntesis es utilizado por la autora como lo hace el materialismo histórico, es decir, no como un resumen sino como un intento de articulación contradictoria entre las diferentes formas de la cultura. En él diferentes sectores disputan y negocian por sus intereses porque el currículum es en términos del sociólogo Pierre Bourdieu (1970) un arbitrario cultural en donde se incorporan elementos hegemónicos que resultan valiosos para una sociedad.

Por esto, en los diferentes espacios que ocupa dicho currículum, tanto formales como prácticos, los elementos pertenecientes a otros grupos socioculturales luchan e incluso logran incorporarse. De este modo, consideramos que la educación no es por sí misma reproductora o transformadora; el carácter que adquiere la función social de la educación está estrechamente vinculado con los proyectos socioculturales y políticoeconómicos desarrollados en el seno de la sociedad. 
Es importante entender que el currículum no es solamente sus estructuras formales sino también su desarrollo práctico. Por eso es necesario comprender su devenir en la práctica concreta, contemplar las particularidades de cada lugar o región, como las de nuestra provincia, en donde muchas veces se pueden observar contradicciones entre las jurisdicciones. Entendemos que los proyectos de currículum se conforman a través de la lucha de distintos sectores sociales lo que produce que la síntesis no solo contenga símbolos hegemónicos sino también de diversos grupos. Es en este marco donde cobra importancia atender la representación que se hace de las comunidades indígenas y preguntarse qué lugar tienen en la educación.

Para pensar una educación y un currículum más amplio e inclusivo rescatamos la noción de justicia curricular (Connell, 2009) que implica garantizar una justicia social, en donde haya currículums que reflejen los intereses de los menos favorecidos. Compartimos la idea de Connell, ya que los currículos separados y diferentes tienen cierto atractivo, pero dejan intacto el currículum hegemónico en vigor, sin cuestionarlo, re-construirlo y a su vez deconstruirlo. El currículum requiere cambiar el punto de partida de forma que encarne los intereses de las personas excluidas.

Entendemos que no existe un único proyecto contrahegemónico. La ciencia social y su praxis actual reconoce una serie de modelos principales de desigualdad: género, clase, raza, etnia, y a escala mundial región y nacionalidad. Por esto, la igualdad no puede ser estática; siempre se está produciendo en mayor o menor grado. Los efectos sociales son dinámicos, por ende, el currículum y la noción de igualdad debe analizarse en relación con las producciones históricas.

En este sentido, consideramos que "la sexualidad estaba y está en todas partes porque es una dimensión de la construcción de la subjetividad que trasciende ampliamente el ejercicio de la genitalidad o una expresión de la intimidad" (Morgade, 2011, p.10). Es necesario desvincularla de las materias o de los contenidos más biologicistas, de las ciencias naturales. La educación sexual debe tener una mirada amplia y transversal porque, tanto la escuela como la institución y todo lo que ocurre en ella, enseña nociones relacionadas con el género y la sexualidad.

Es por esto que reconocemos como primordial la dimensión de género vinculada a todas las disciplinas y a todos los niveles sin excepciones. No obstante, debemos tener en cuenta que no siempre la educación sexual es integral, con enfoque de géneros y de derechos humanos. Resulta imprescindible reconocer la desigualdad en torno a esta categoría, no solo desde lo institucional y/o académico sino también en todo lo que sucede dentro de los espacios educativos. 


\section{Marcos normativos: Interculturalidad, Ley de} Educación Sexual Integral (ESI) y lineamientos curriculares de Santa Fe

La normativa de nuestro país plantea lineamientos de los cuales es necesario hacer mención. Por un lado, en el panorama internacional, Argentina adhiere a los fundamentos y artículos de derecho internacional propuestos por la Organización de las Naciones Unidas (ONU) y la Organización de los Estados Americanos (OEA). Rescatamos de estos tratados el siguiente artículo:

Deberán adoptarse medidas de carácter educativo en todos los sectores de la comunidad nacional, y especialmente en los que estén en contacto más directo con los pueblos interesados, con objeto de eliminar los prejuicios que pudieran tener con respecto a esos pueblos. A tal fin, deberán hacerse esfuerzos por asegurar que los libros de historia y demás material didáctico ofrezcan una descripción equitativa, exacta e instructiva de las sociedades y culturas de los pueblos interesados (Convenio No169 OIT sobre Pueblos Indígenas y Tribales, 2014, p.130)

Desde el año 2006, la normativa nacional de nuestro país en el ámbito educativo es la Ley de Educación Nacional N²6.206. En la misma se plantea la necesidad de cuestionar a los modelos tradicionales de los contenidos educativos que tienden a la homogeneización, sumando la propuesta de la interculturalidad. Esta ley garantiza una educación intercultural bilingüe en los niveles inicial, primario y secundario. A su vez, el artículo 54 plantea que

El Ministerio de Educación, Ciencia y Tecnología, en acuerdo con el Consejo Federal de Educación, definirá contenidos curriculares comunes que promuevan el respeto por la multiculturalidad y el conocimiento de las culturas originarias en todas las escuelas del país, permitiendo a los/as alumnos/as valorar y comprender la diversidad cultural como atributo positivo de nuestra sociedad (Ley de Educación Nacional 26.206, 2006).

Esta normativa nos invita a reflexionar acerca de cuán importante es sumar contenidos que fomenten el conocimiento y el respeto por la multiculturalidad tanto desde el derecho de las comunidades originarias como también de toda la sociedad con el fin de fomentar la diversidad cultural.

Además, en nuestro país desde octubre de 2006 se creó la ley 26.150 que crea el programa de educación sexual integral donde se cambian los lineamientos de la política educativa y se lleva este enfoque a los distintos niveles del ámbito escolar. Con esta legislación la ley busca garantizar los derechos de les estudiantes tanto en gestión estatal como privada. Los contenidos deben respetar la diversidad sexual, 
reconocer la perspectiva de género, cuidar el cuerpo y la salud. A su vez, articular los contenidos de ESI a la realidad sociocultural.

Sin embargo, además de la normativa nacional es necesaria la incorporación desde el sistema provincial. Por esto en mayo de 2018, el Consejo Federal de Educación, que reúne al conjunto de los Ministerios de Educación del país y del cual Santa Fe es parte, a través de la Resolución 340/18 señaló que

con el objeto de dar efectivo cumplimiento a la Ley 26.150 de Educación Sexual Integral y garantizar el desarrollo de los lineamientos curriculares, las jurisdicciones se comprometen a implementar la obligatoriedad de la educación sexual integral en todos los niveles y modalidades educativas, abordando, sin excepción, los cinco ejes conceptuales: Cuidar el cuerpo y la salud; Valorar la afectividad; Garantizar la equidad de Género; Respetar la diversidad y Ejercer nuestros derechos (Anexo Resolución del CFE N $\mathrm{N}^{\circ} 340 / 18,2018$, p.5).

Así a nivel provincial mediante la ley No 38685 se establece, en su artículo 4, que la Educación Sexual Integral tiene una serie de objetivos, dentro de los cuales rescatamos "promover la igualdad de género y el respeto de la diversidad en sus múltiples manifestaciones como constitutiva de la condición humana, como así también al multiculturalismo" (Proyecto de Ley No 38.685, 2020, p.2).

Además, debemos tener en cuenta el Diseño Curricular de Educación Secundaria Orientada, donde el contenido obligatorio está basado en una serie de ejes temáticos, por ejemplo, el análisis en torno a las formas de organización de las sociedades indígenas americanas, la transición del mundo feudal a la Modernidad; la comprensión de las causas múltiples e interrelacionadas de la expansión ultramarina europea; la comprensión de los procesos de conquista europea en América desde interpretaciones múltiples; el estudio analógico del proceso de colonización con particular referencia a Hispanoamérica; la comprensión de los cambios culturales que conlleva la colonización en Latinoamérica; problematizando en torno a las categorías de aculturación, deculturación, transculturación; el análisis de los cambios del sistema colonial hispanoamericano.

Con base a estas normativas, cada alumne debe poder desarrollar y afianzar actitudes, saberes y prácticas que le sirva para promover actitudes responsables con la diversidad cultural, la no discriminación y el reconocimiento de hechos históricos de su propio territorio con especial énfasis en la importancia de la historia de las mujeres. En la búsqueda de este fin, consideramos relevante el hallazgo de la Dama de Cao porque atraviesa el contenido hegemónico y deconstruye desde una perspectiva de 
género los roles sexo-género permitiendo considerar experiencias de culturas extraeuropeas.

\section{Caso Mochica: ¿quién es la Dama de Cao?}

En la costa norte peruana se desarrollaron culturas diversas, entre 1500 a.C y 500 d.C. surgieron las primeras civilizaciones. Todas las culturas arqueológicas previas fueron sintetizadas en lo que posteriormente fue la cultura Mochica. Los trabajos arqueológicos en Perú en la región El Brujo comenzaron a finales de la década de 1980. No obstante, el salto a la fama de Magdalena de Cao se produce cuando un grupo de arqueólogos peruanos en los años 2004 y 2005, encabezado por Régulo Franco, descubren en el interior de una tumba el cuerpo de una mujer de mediana edad que habría vivido en los albores de la cultura Mochica en el siglo IV d.C y la llamaron la Dama de Cao.

Este hallazgo arqueológico es único en su clase en Abya yala ya que por primera vez se registró un ajuar funerario de gran impronta asociado a una mujer. Según la evidencia arqueológica el especial mausoleo en donde fue encontrada la Dama de Cao conservaba en su interior tanto iconografía marítima como terrestre.

En el recinto ceremonial del hallazgo la Dama de Cao fue encontrada conservada en repelente de cinabrio o sulfuro de mercurio, lo cual permitió hallarla en un muy buen estado. Actualmente, para garantizar la debida preservación de su cuerpo a futuro se ha producido una réplica de esta invalorable evidencia arqueológica mediante tecnología 3D para promover su observación y estudio para el beneficio de las futuras generaciones.

Alrededor de la tumba de la Dama de Cao, estaban sepultadas lo que se cree son sus pertenencias personales: diadema de oro, vestidos, aretes, collares, mantas de algodón, entre otras. Asimismo, en el exterior del mausoleo se encontraba acompañada de individuos sacrificados recurrentes en la iconografía mochica.

Según la evidencia arqueológica el especial mausoleo en donde fue encontrada la Dama de Cao conservaba en su interior tanto iconografía marítima como terrestre. El río y el mar eran fuentes primordiales de subsistencia lo que refleja la cosmovisión mítica y geográfica mochica. En relación,

el mar que baña las costas andinas es el punto de conexión entre la divinidad marina y el desarrollo político-religioso femenino en la costa norte del Perú. Desde los primeros estudios andinos, el ámbito marítimo es considerado un espacio sagrado y, a la vez, una fuente de abastecimiento de recursos para el mantenimiento del grupo humano. El mar es considerado como la madre de todas las aguas (Escudero, 2015, p.9). 
En las iconografías marítimas de la cultura moche se vinculaba tanto al mar como a animales marítimos en escenas de rituales donde la presencia femenina implicaba una sacralidad ligada al espectro político por medio de la espiritualidad.

Cada elemento del recinto ceremonial en el que fue hallada, desde la elección de lugar, los acompañamientos funerarios, piezas y objetos del mausoleo y la forma de preservación de su cuerpo son resultado de la toma de decisiones que nos ayudan a descubrir quién era y su identidad.

La Dama de Cao presentaba tatuajes en sus brazos y cuerpo, figuras de arañas y serpientes, animales vinculados con la fertilidad de la tierra y el agua, que formaban parte del imaginario religioso. Estos tatuajes visibilizan la impronta política que debió haber tenido. Por otro lado, probablemente debió ser una sacerdotisa en los rituales dedicada a actividades espirituales muy profundas, asociadas al curanderismo, propio de la cultura mochica.

El tipo de enterramiento evidencia que la Dama de Cao pertenecería a un grupo relevante de poder. Los honores detrás de cada pieza y objeto colocados al interior y al exterior de su tumba reflejan que estaba vinculada al estrato más alto de la sociedad. Sin embargo, debemos tener presente que estas sociedades no se estructuraban de la misma manera que las sociedades modernas por lo cual es complejo clasificar los límites por los cuales se diferencian estas esferas y cómo se hallaban articuladas. Lo que sí podemos vislumbrar es una fuerte importancia del aspecto religioso permeando las demás esferas.

Según las investigaciones realizadas en el Complejo Arqueológico El Brujo por la Fundación Wiese y FARO Technologies, por medio de la antropología física, se estima que murió producto de un embarazo o posterior a este acto de acuerdo con las características de su vientre. No obstante, hay incógnitas al respecto que nos lleva a pensar cómo podríamos resaltar este aspecto, ¿cuán separado estaba la maternidad de la función política? La verdad que esto no podemos saberlo, aunque podemos suponer que este aspecto no debía estar separado de la vida social, ya que la esfera de lo político y lo público no eran entendidas como en la actualidad, sino más bien existe una complementariedad simbólica y ritual.

Los arqueólogos Luis Jaime Castillo Butters y Carlos E. Rengifo Chunga reflexionan a través de la interpretación iconográfica en la cerámica acerca del rol e identidad de las mujeres en la comunidad mochica tardía en San José de Moro ya que aquí podemos suponer que la Dama de Cao generó influencias en otras mujeres. Se pudo observar que en períodos posteriores el papel de la mujer en ámbitos de lo sagrado y sobrenatural fue en aumento como es el caso de las Sacerdotisas de San José de Moro en el sector mochica norte. Aquí las representaciones iconográficas 
reflejan que las mujeres se hallaban presentes tanto en actividades domésticas como rituales ceremoniales.

Primero fue el Señor de Sipán, que fue asociado con un personaje sobrenatural masculino, ataviado como guerrero, que preside la Ceremonia del Sacrificio, donde recibe una copa con la sangre obtenida de prisioneros vencidos en batallas rituales (Alva y Donnan 1993). Luego siguieron las mujeres encontradas en las tumbas de cámara en San José de Moro, que fueron asociadas con las Sacerdotisas míticas, que aparecen en la representación iconográfica de la Ceremonia del Sacrificio presentando una copa o también en balsas cargadas de ofrendas y prisioneros para los rituales de sacrificio (Castillo y Rengifo, 2008, p.5).

Es en este último aspecto de participación ritual donde aparecen activamente representadas dando cuenta de que el género no era un limitante para realizar ciertas experiencias $\mathrm{y} / \mathrm{u}$ ocupar espacios de poder. Las representaciones femeninas predominan en todas las escenas sobrenaturales y rituales.

Consideramos que muchos de los estudios e investigaciones han extrapolado una lógica de género actual, por ende necesitamos replantearnos los modos y formas con los cuales nos acercamos a estas interpretaciones. En la interpretación del pasado no debemos juzgar necesariamente que se hayan diferenciado los roles de género acentuadamente como hoy en día o que hayan tenido caracterizaciones específicas de sexo-género ya que pertenecen a otra coyuntura con perspectivas y particularidades propias. Por ello, es de gran relevancia los lentes o la lupa con que se interpele a las colectividades a analizar.

Transformar y concebir modos de enseñar distintos a lo inculcado e impuestos socialmente no es una tarea sencilla. Sugiere la continua interpelación a lo dado y establecido. Implica buscar nuevos conceptos, herramientas, maneras de conocer, problemáticas que atraigan e interroguen, creando nuevas sensibilidades porque el proceso educativo no es un contenido cerrado y estático es una construcción colectiva, un campo abierto por y para todes, tanto alumnes como profesores. En este sentido,

debemos ser conscientes de que la escuela es un ámbito de socialización y a la vez de disciplinamiento sobre los cuerpos, en la cual se transmiten ideas y prácticas, estereotipos de género, distintas expectativas ya se traten de alumnos o alumnas, ideas de "normalidad", silencios que invisibilizan y deslegitiman a las mujeres y a la disidencia sexual como parte de los contenidos culturales de la sociedad en la que la escuela está inserta (Argiroffo y Scalona, 2016, p.29). 
Tanto en la fundamentación como en el contenido educativo del ciclo básico como orientado muchas veces lidiamos con enfoques que no plantean ninguna perspectiva de género. Orientaciones formativas en donde se invisibiliza históricamente a las mujeres y se reproduce así una única mirada posible: la masculina. Además, esto implica presentar al hombre como el único protagonista lo que le quita participación a la mujer.

En los lineamientos del ciclo básico y orientado de Ciencias Sociales, la ley busca repensar las nociones en torno al género reconociéndose como constructos socio históricos y promueve la diversidad de intereses y particularidades culturales y étnicas. Es por esto que asumimos activamente la responsabilidad política de para qué, para quiénes y cómo enseñamos. Es en este marco en donde consideramos esencial la divulgación del hallazgo de la Dama de Cao porque forma parte de un proceso de resignificación histórica para las comunidades, en particular, y para el mundo.

¿Qué importancia tendría articular el caso de la Dama de Cao en el proyecto de escuela que deseamos? Esto radicaría en la importancia en que se instala en les alumnes una conciencia del papel histórico de las mujeres indígenas en los diferentes procesos sociales que conviven en luchas actuales por derechos de las mujeres de las comunidades aún en disputa.

En este sentido, para pensar los saberes en relación con la transformación de la realidad, reflexiona acerca del rol de les educadores y les alumnes.

(...) pensar acertadamente impone al profesor o, en términos más amplios, a la escuela, el deber de respetar no sólo los saberes con que llegan los educandos, sobre todo los de las clases populares saberes socialmente construidos en la práctica comunitaria-, sino también, como lo vengo sugiriendo hace más de treinta años, discutir con los alumnos la razón de ser de esos saberes en relación con la enseñanza de los contenidos (Freire, 2008, p.10).

También es importante repensar y comprender los significantes de las comunidades ya que tienen sus propios diálogos y saberes. Cuando se ven incorporados a una enseñanza tradicional chocan con conocimientos que no forman parte de sus saberes construidos lo que genera una doble forma de exclusión ya que las comunidades no pueden sentirse cómodas dentro de las instituciones con sus currículums y porque quienes están ajenos a sus problemáticas se les veta el derecho a conocer otros saberes culturales. Como consecuencia, acentúa la otredad y la discriminación hacia los pueblos indígenas. 
En relación con ello, podemos tener en cuenta el método de Freire, el dialogal, no solo como una forma de dar clases sino también desde las instituciones educativas hacia las comunidades indígenas.

En este sentido,

(...) el derecho a acceder a la educación, el derecho como contenido de la educación, el derecho a un trato no prejuicioso en las instituciones educativas y la adaptación de los contenidos a las necesidades de los diversos sujetos colectivos, demandan la descolonización de escuelas y universidades. Es posible que el primero de todos ellos, si es debidamente implementado, pueda reforzar y hasta garantizar los otros tres. La discriminación, el eurocentrismo y la alterofobia vigentes en las escuelas y en las universidades han reservado la entrada y el buen tránsito por el proceso educativo para las élites y sus colaboradores y, con esto, también permitieron el control monopólico de las profesiones y de las narrativas de los grandes temas nacionales (Segato, 2015, p.293).

La pulseada con el saber hegemónico, con el eurocentrismo y la discriminación continúa vigente por eso llamamos a generar una pedagogía militante al servicio de les estudiantes y de los derechos humanos. Sabemos que las respuestas sin duda son variadas y complejas. Pero cualesquiera que sean no podrán evadir la tensión de inventar nuevos vocabularios que permitan a las antiguas y nuevas generaciones reencontrarse con los viejos y nuevos problemas.

\section{Conclusiones}

Por medio de esta presentación se intentó, desde una mirada crítica y no convencional, generar una divulgación acerca del caso específico de la Dama de Cao, como contenido educativo para repensar la mirada con la cual se ha enseñado e investigado a las mujeres indígenas en la historia y en particular cómo hemos visto la historia antigua en Perú.

Creemos que la Dama de Cao es un buen recurso para llevar a las aulas e incursionar en nuevos hallazgos e investigaciones actuales para dar a conocer a las mujeres indígenas, repensar la exclusión de las mujeres en la historia a través de nuevos interrogantes, interpelar al pasado y el presente e incorporarlo en el currículum a modo de tensionar problemáticas actuales.

En relación con esto también podemos pensar en un caso regional a trabajar como por ejemplo La Delfina, para articular similitudes y diferencias a nivel continental y temporal. Alentamos a profundizar e investigar otros casos posibles para incorporar en el espacio educativo, la historia que buscamos transmitir tiene que ver con la ruptura y la búsqueda de otros relatos no convencionales. A su vez, la lógica de la enseñanza de la historia dentro de la escuela secundaria responde al 
ordenamiento de lo que conocemos como Historia Universal que se nos presenta como única. Este caso nos permite tensionar y matizar desde la historia latinoamericana para poder rescatar otras voces invisibilizadas y excluidas que nos permiten profundizar un registro macro.

Además, debemos considerar que es necesario, en la medida de lo posible analizar la realidad de las mujeres indígenas con relación a otras y su rol de la sociedad y no caer en biografías o en casos unipersonales. En lo que respecta a la Dama de Cao es complejo compararla con otras mujeres ya que en la historia antigua faltan fuentes o todavía no se han encontrado. Pero sí podemos pensarla con relación a lo que conocemos de la cultura Moche.

Concluimos en que es necesario complejizar el enfoque de los procesos históricos, así como los actuales, que se han centrado en dominantes y dominades, dando prioridad al punto de vista de les primeres, es por ello por lo que consideramos de suma importancia repensar cómo es y fue esta relación, poniendo el foco en los márgenes para así dar voz a les excluides de la historia. Esto no significa crear una historia exclusiva de un determinado colectivo excluido, o de varios, en modos separados, sino que consideramos que el desafío consiste en no centrarnos exclusivamente en les marginades, haciendo solo una historia de mujeres o solo una historia indígena, lo que se intenta es de comprender y deconstruir esas relaciones de dominación para poder crear nuevos vínculos entre estos grupos sociales.

Es ancladas en este presente violento hacia las mujeres y al colectivo indígena que nos convoca con urgencia. Nos vemos movilizadas a resignificar un pasado histórico, a cuestionarnos el tipo de ciencia y educación que estamos produciendo y reproduciendo ya que constantemente se piensa a los pueblos indígenas en el pasado y no se consideran sus problemáticas presentes y la vulneración de sus derechos, se deja un vacío académico y educativo sobre la propia comunidad.

Las instituciones educativas incurren en representar al pasado indígena como parte de una historia que no dialoga con el presente como si ya no existieran y perpetuando en el imaginario un estereotipo del indígena que no contribuye a entender sus propias prácticas actuales.

Consideramos que debemos crear espacios y formas para garantizar sus derechos y reivindicaciones desde una construcción colectiva donde les excluides tengan un rol protagónico. Nos parece que este proceso es más amplio y se extiende a todas las luchas y reivindicaciones del colectivo indígena en la actualidad. Donde ellos mismos puedan contar sus historias, mantener vivas sus lenguas y culturas con la misma legitimidad que la cultura occidental posee en nuestra sociedad. 
A modo de cierre, invitamos a realizar este recorrido no tradicional para disputar los discursos comunes sobre las comunidades indígenas regionalmente ya sea desde nuestro rol tanto como docentes, investigadores y ciudadanes con la convicción de que el conocimiento y los interrogantes en torno al pasado y realidad de las mujeres indígenas construiría nuevas realidades sin violencias y con mayores derechos.

\section{Referencias bibliográficas}

Argiroffo, B. y Scalona, E. (eds). (2016). Educación Sexual Integral y enseñanza de las Ciencias Sociales. Enfoques, sujetos y objetos para el abordaje en la escuela secundaria. Rosario: AMSAFE-COAD.

Bourdieu, P. y Passeron, J, C. (1970). La reproducción. Elementos para una teoría del sistema educativo. París: Minuit.

Castillo, L. J. y Rengifo, C. (2008). Identidades funerarias femeninas y poder ideológico en las sociedades mochicas. En K. Makowski (ed), Los señores de los reinos de la luna, Colección de Arte y Tesoros del Perú. Lima: Banco de Crédito del Perú.

Connell, R, W. (2009). La justicia curricular. Buenos Aires: Laboratorio de Políticas Públicas.

Consejo Federal de Educación. Resolución CFE N³40/18. Misiones, 22 de mayo de 2018.

Contreras H, P y Cristoffanini T, M. (2016). Desde las epistemologías feministas a los feminismos decoloniales: aportes a los estudios sobre migraciones. Barcelona: Universidad de Barcelona.

Convenio Núm. 169 de la OIT sobre pueblos indígenas y tribales en países independientes. Declaración de las Naciones Unidas sobre los Derechos de los Pueblos Indígenas. Lima: OIT/Oficina Regional para América Latina y el Caribe, 2014.

De Alba, A. (1995). Currículum: crisis, mito y perspectivas. Buenos Aires: Miño y Dávila editores.

Diseño Curricular de Educación Secundaria Orientada. Ministerio de Educación de la Provincia de Santa Fe, 2014.

Escudero, A, A. (2015). Sacerdotisas, Curanderas, Parteras y Guerreras: Mujeres de élite en la costa norte del Perú Antiguo. Revista de Estudios Latinoamericanos de la Universidad Pablo De Olavide De Sevilla.

Freire, P. (2008). Pedagogía de la Autonomía: Saberes necesarios para la práctica educativa. Editorial SXXI. 
Lander, E. (2000). La colonialidad del saber: eurocentrismo y Ciencias Sociales. Perspectiva Latinoamericana. Buenos Aires: FLACSO.

Ley de Educación Nacional, Ley $\mathrm{N}^{\circ}$ 26.206,14 de diciembre, 2006.

Ministerio de Educación Nacional (2008). Programa Nacional de Educación Sexual Integral Ley Nacional N.o 26.150, Lineamientos Curriculares para la Educación Sexual Integral. Buenos Aires.

Morgade, G. (2011). Toda Educación es sexual: hacia una educación sexuada justa. Buenos Aires: La Crujía.

Proyecto de Ley No 38685. Cámara de Diputados de Santa Fe, 9 de septiembre de 2020.

Santos, Boaventura de Sousa. (2010). Descolonizar el saber, reinventar el poder. Uruguay: Editorial Trilce.

Segato, L, R. (2015). La crítica de la colonialidad en ocho ensayos. Y una antropología por demanda. Buenos Aires: Ed. Prometeo.

Scott, J, W. (1990). El género: una categoría útil para el análisis histórico. En J. Amelang y M. Nash (eds), Historia y género: las mujeres en la Europa moderna y contemporánea. Valencia: Institucio Valenciana d'estudis i investigación. 